\title{
O movimento ocular na leitura realizada por revisores de textos
}

\section{Revisers' eye movement in reading}

Délia Ribeiro Leite

Conselho Regional de Contabilidade de Minas Gerais, Belo Horizonte, Minas Gerais

/ Brasil

dribeiroleite@yahoo.com.br

José Olímpio de Magalhães

Universidade Federal de Minas Gerais, Belo Horizonte, Minas Gerais / Brasil joseolimpiomagalhaes@yahoo.com.br

Resumo: Este estudo objetivou investigar o processamento da leitura de profissionais revisores de textos, comparando o movimento ocular destes com o de sujeitos que não trabalham profissionalmente com revisão (não revisores). Na tarefa de leitura e detecção de erros realizada por 14 revisores e 14 não revisores, os participantes deveriam ler e revisar pequenos textos jornalísticos projetados no computador, clicando com o mouse nos erros e/ou inadequações que encontrassem. Havia dois tipos de erros nos estímulos experimentais: a) supressão de preposição e b) incoerência gerada por uma anáfora nominal incorreta. $\mathrm{Na}$ análise estatística, quando garantida a normalidade, foram realizados modelos mistos; do contrário, a análise foi realizada por meio de testes não paramétricos. Quanto à análise do movimento ocular, a perspectiva geral foi de que os revisores apresentaram valores mais elevados, o que significa que eles foram mais lentos na leitura do que os não revisores, o que foi identificado nas medidas do nível do texto, da sentença e do trecho alvo. Esta pesquisa contribui para uma caracterização dos processos de leitura envolvidos em tarefas de revisão de textos por revisores profissionais.

Palavras-chave: revisão de textos; revisão profissional; movimento ocular; proficiência. 
Abstract: This study aims to investigate the reading processing of professional revisers comparing their eye movement with subjects who do not work professionally in revising (non revisers). In the reading and error detection task proposed and performed by 14 revisers and 14 non revisers, the participants had to read and edit short journalistic texts displayed on a computer, by clicking on the errors and/or inadequacies they found using the mouse. There were two types of errors: a) suppression of preposition and b) incoherence generated by an incorrect nominal anaphora. In the statistic analysis, when normality was ensured, mixed models were carried out; otherwise, the analysis was carried out by means of non-parametric tests. Considering the eye movement analysis, the general perspective indicate that revisers presented the highest values, meaning they were slower in reading than non revisers, which occurred concerning the measures of the text, sentence and local levels. This research contributes to a characterization of reading processes involved in revisions of texts by professional reviewers.

Keywords: revision; professional revisers; eye movement; proficiency.

Recebido em: 9 de dezembro de 2016.

Aprovado em: 9 de janeiro de 2017.

\section{Introdução}

A revisão está diretamente relacionada com o processo de leitura e produção de textos. Por isso, ao relermos um texto que produzimos, buscamos assumir uma visão exotópica ${ }^{1}$ e verificar aspectos diversos da estrutura textual, de forma a identificar possíveis erros, bem como trechos cuja interpretação seja difícil para o leitor. No entanto, nem sempre o autor é o melhor leitor para revisar o seu próprio texto, seja por questões técnicas, relativas à falta de conhecimento específico no campo dos estudos da linguagem (no caso de autores de outras áreas), seja por questões práticas: a exotopia pode ficar comprometida, tendo em vista que o texto, ao ser lido pelo próprio autor, perde a imprevisibilidade, o que pode fazer com que as inadequações de linguagem não sejam percebidas.

\footnotetext{
${ }^{1}$ A visão exotópica é aquela em que a leitura é realizada com um olhar diferente daquele do produtor do texto, um olhar que simularia a leitura realizada pelo leitor para o qual aquele texto é destinado.
} 
Por isso, há o profissional revisor de textos, cuja ocupação é a leitura dos textos produzidos por outrem, de forma a identificar inadequações de linguagem e propor correções ou alterações diversas.

Apesar de ser uma atividade antiga e importante, poucos são os estudos cujo objeto é a revisão profissional, muito embora haja um imaginário de que essa tarefa exige uma demanda e uma qualificação especiais, principalmente no que se refere à leitura: "O revisor não lê como os demais homens leem, ele fotografa a palavra visualmente; e o texto reflete-se em sua correção." (WAGNER; CUNHA, 2012, p. 12).

Dessa forma, este trabalho propõe-se a investigar o processamento da leitura realizada por profissionais revisores de textos. Para tanto, foi utilizado como método de investigação o rastreamento ocular. Verificamos os padrões de fixações e sacadas realizados por esses profissionais durante a revisão, bem como se esses padrões se aproximaram do depreendido quando indivíduos que não trabalham profissionalmente com revisão leem os mesmos textos. Portanto, este trabalho tem como objetivos:

- Investigar os padrões do movimento dos olhos na leitura realizada por profissionais revisores de textos, em uma tarefa de detecção de erros;

- Comparar o movimento ocular de profissionais revisores de textos com o de sujeitos que não realizam profissionalmente a revisão, em uma tarefa de leitura e detecção de erros;

- Verificar se os profissionais revisores de textos e se os sujeitos que não realizam a revisão profissional fazem uma leitura voltada tanto para a superfície textual quanto para níveis mais globais do texto.

A hipótese assumida neste estudo é a de que a leitura realizada pelos revisores de textos é menos automática, mais controlada e mais detalhada, o que acarreta mais proficiência na atividade de revisão.

\section{A revisão de textos sob uma abordagem cognitiva}

Heurley (2006) destaca três principais visões da revisão na perspectiva cognitiva: a revisão como uma modificação efetiva de um texto; a revisão como subprocesso do processo de escrita, visando melhorar o texto já escrito; e a revisão como componente distinto da 
produção escrita, que envolveria um conjunto de subprocessos e de procedimentos implicados no controle da escrita.

Hayes et al. (1987), por sua vez, propõem um modelo especificamente de revisão. $\mathrm{O}$ modelo é dividido em processos e conhecimentos. O primeiro processo envolvido é a definição da tarefa, que abarca questões como os objetivos do revisor, os traços do texto a serem examinados e a forma como se pretende proceder à revisão. O segundo é a avaliação, quando são selecionados os objetivos da leitura: compreensão, avaliação ou detecção de problemas. Com base na representação que se faz do problema, será selecionada uma estratégia, sendo possível: modificar ou controlar o processo de revisão em si ou modificar o texto. Ao modificar o processo de revisão, o revisor pode ignorar o problema, buscar mais informações de forma a melhorar o seu diagnóstico ou adiar a ação, inclusive quando opta por fazer mais de uma leitura, uma delas direcionada a um nível mais alto e outra relacionada com os aspectos da superfície textual. Ao contrário, se opta por modificar o texto, o revisor pode reescrevê-lo ou revisá-lo. Nesse caso, a revisão comporta os casos em que o revisor corrige as inadequações mantendo o máximo possível do texto original. Uma questão importante é que os revisores podem redefinir a tarefa ao longo da realização da revisão e, assim, o conhecimento utilizado, que inclui objetivos, critérios e restrições, é modificado dinamicamente durante a revisão. A revisão está relacionada com a leitura para detecção de problemas, que, de acordo com os autores, é diferente, por exemplo, daquela direcionada somente à compreensão.

Em 1996, Hayes propõe uma reformulação do modelo de escrita de Hayes e Flower (1980), com o objetivo principal de acrescentar elementos cognitivos importantes, como a memória de trabalho, assim como reorganizar os elementos existentes de forma a mostrar a relação deles com processos cognitivos mais gerais envolvidos em vários tipos de atividades. A revisão passa a ser vista como uma forma de interpretação de texto. O autor propõe um novo modelo de revisão, no qual há uma estrutura de controle, composta pelo esquema da tarefa de revisão; processos fundamentais, entre eles a reflexão, o processamento e a produção do texto; e os recursos utilizados, que são a memória de trabalho e a memória de longa duração. A revisão integra juntamente com a reflexão e a produção do texto o subcomponente dos processos cognitivos, o qual, por sua vez, está inserido em um componente maior. 
Este, além dos processos cognitivos, envolve um subcomponente afetivo/ motivacional, a memória de trabalho e a memória de longo prazo. Assim, o autor considera que, para entender a revisão, é necessário entender não só o processo, mas também sua estrutura de controle e os recursos envolvidos. $\mathrm{O}$ autor pontua que esse conjunto é adquirido com a prática, o que se depreende, inclusive, da diferença entre revisores e produtores de textos experientes e novatos explorada em Hayes et al. (1987).

Como se vê, no âmbito desses estudos, a revisão deixou de ter um papel secundário no processo de escrita e passou a ser vista como uma etapa importante desse processo, que atua em vários níveis. Cada vez mais, ela tem sido tratada como uma atividade de controle, que pode operar separadamente ou ao longo do processo de escrita.

\section{Movimento ocular e leitura}

Os olhos se movimentam em sacadas intercaladas com fixações. Enquanto a sacada corresponde a um rápido movimento dos olhos para mover o foco de uma área para outra, a fixação equivale ao tempo gasto focalizando-se uma determinada área. É possível a verificação de variáveis temporais e espaciais quando se registra o movimento dos olhos, já que há um deslocamento espacial, ou seja, para onde será direcionado o movimento, e um deslocamento temporal, representado pelo momento em que ocorrerá esse movimento. Além disso, sabe-se que o tipo de movimento depende bastante da informação requerida (LAND, 2007, p. 78).

Além disso, nem sempre o movimento ocular ocorre de maneira linear, pois há situações em que são realizadas regressões para locais que já foram focalizados. De acordo com Luegi (2006, p. 23), "Cerca de 15\% dos movimentos sacádicos durante a leitura são movimentos regressivos, ou seja, partem da direita para a esquerda, para regiões anteriores do texto, na mesma linha ou em algumas linhas acima".

As regressões podem ser correções de sacadas que foram muito longas e, em função disso, é necessário que os olhos façam um breve retorno. Podem, também, ser decorrentes de dificuldades de entendimento de alguma parte do texto. Nesse caso, geralmente, as regressões são longas (maiores que 10 caracteres na mesma linha ou em direção a linhas anteriores) e o comportamento com relação ao retorno varia conforme a proficiência do leitor. 
O registro do movimento dos olhos é utilizado em uma grande gama de estudos sobre o processamento da linguagem. Muitas variáveis podem influenciar os valores das fixações e das sacadas, inclusive se se trata de uma leitura em voz alta ou silenciosa. Como não há estudos específicos sobre o movimento ocular durante a revisão de textos, nesta seção, serão apresentados estudos que abordam questões relacionadas aos tipos de erros presentes nos textos experimentais deste trabalho, bem como fatores considerados na análise depreendida neste trabalho, como os retornos e a releitura dos textos.

Como o movimento dos olhos se processa em fixações e sacadas, de uma fixação para outra podem ser puladas palavras. Sabese que palavras funcionais tendem a ser puladas, ou seja, não serem focalizadas. De acordo com Rayner, as palavras funcionais são fixadas aproximadamente 35\% das vezes (RAYNER, 1998, p. 375). Isso pode ser ocasionado por elas serem mais facilmente identificáveis, ou seja, mais previsíveis pelo contexto, ou mesmo pelo fato de elas serem mais frequentes (STAUB; RAYNER, 2007). O fato de elas serem curtas também pode influenciar, já que, à medida que o tamanho da palavra aumenta, a probabilidade de ela ser fixada também aumenta - palavras de 2 a 3 letras são fixadas, em geral, 25\% das vezes (RAYNER, 1998, p. 375).

Hyönä e Nurminem (2006) investigam as regressões e, em seus resultados, os leitores que tendem a direcionar as regressões para partes realmente informativas do texto conseguem apresentar um resumo melhor daquilo que leram. Dessa forma, há também aspectos relacionados a níveis textuais mais altos que interferem no movimento dos olhos. Os autores pesquisam como leem leitores competentes, com o objetivo de confirmar a classificação proposta em estudo anterior (HYÖN̈̈; KAAKINEN; LORCH, 2002) e verificar se os próprios leitores têm consciência das estratégias de leitura que eles utilizam. Foram identificados três padrões de leitores: os processadores de estruturas de tópico, que foram minoria, seguidos dos leitores lineares rápidos e, por fim, dos leitores lineares lentos, que foram maioria. Os leitores têm consciência do tempo demandado na leitura, se são ou não lentos, bem como sabem se fazem ou não retornos a partes do texto, porém não têm uma noção precisa de quais os locais para os quais eles fazem retornos. Portanto, há diferenças idiossincráticas relacionadas ao movimento ocular e à leitura, embora o direcionamento dado na tarefa possa influenciar os padrões. No caso da 
pesquisa acima, os leitores tiveram que fazer um resumo do texto, o que pode ter influenciado a maneira como eles se comportaram.

Hyönä e Niemi (1990) investigam a releitura de um texto. A releitura faz com que o processo seja facilitado e, com isso, todas as medidas investigadas são diminuídas, tanto o número e o tempo de fixações quanto o número de regressões. Os autores também investigam se a releitura influencia na tendência em se fixarem mais os trechos com informações importantes. Nesse sentido, a conclusão é de que as partes mais importantes tendem a ser mais fixadas, mas isso ocorre tanto na primeira leitura quanto na releitura. No entanto, a releitura facilita o processamento das sentenças que são mais informativas do que daquelas não informativas. Além disso, para cada leitura sucessiva, a média das fixações é mais longa no início dos textos do que no final deles.

Vauras, Hyönä e Niemi (1992) investigam a leitura de textos coerentes e incoerentes. Nos textos incoerentes, há a mudança da ordem das sentenças. Os autores utilizam como metodologia o registro do movimento dos olhos e também uma medida off-line, que é a análise de reescritas dos textos realizadas pelos leitores. Eles concluem que incoerências aumentam tanto o tempo de fixação quanto as regressões. No entanto, embora em textos coerentes o aumento do tempo de fixação ocasione melhora na reescrita da parte fixada, o mesmo não ocorre com textos incoerentes.

Luegi (2006) pesquisa o registro do movimento dos olhos entre leitores do português europeu. A autora apresenta sentenças modificadas com a finalidade de se criarem inconsistências (agramaticalidade ou ambiguidade) e verifica a reação dos leitores. Além disso, essas manipulações são realizadas em dois textos: um em que predominam termos técnicos e outro cujo tema é mais comum. A autora verificou que o efeito das variáveis só é visível quando se trata do texto com vocabulário técnico e manipulação das sentenças gerando inconsistências. Esses resultados são vistos tanto no número de fixações quanto no tempo total de leitura. Além disso, a autora verifica que os leitores são bastante precisos no que se refere às regressões e retornam especificamente para o local onde há problemas.

Alamargot et al. (2006) registram concomitantemente o movimento ocular e a execução grafomotora para investigar o processo de escrita. Embora o foco do trabalho não seja a revisão de textos, eles observam que a detecção de erros tipográficos pode ocorrer em tempos 
muito curtos, como o de uma fixação na leitura. Pode, ainda, ocorrer em paralelo com a execução grafomotora.

Portanto, o registro do movimento dos olhos pode indicar aspectos importantes do processamento da leitura. Dessa forma, os trabalhos experimentais demonstram que tanto a fisiologia da visão quanto o processamento da leitura são determinantes quanto ao padrão do movimento dos olhos adotado pelos sujeitos. Esses estudos dão suporte à hipótese deste trabalho de que haverá um padrão de leitura diferenciado conforme o tipo de erro e, também, em função da experiência dos sujeitos em revisão de textos.

\section{Metodologia}

\subsection{Participantes}

Os sujeitos participantes da pesquisa foram divididos em dois grupos: não revisores e revisores, sendo que cada grupo foi composto por 14 sujeitos. No grupo dos não revisores, participaram da pesquisa estudantes de ensino superior que não cursavam Letras nem Comunicação Social, pois a formação nesses dois cursos é pré-requisito para a investidura de vários cargos de revisão e, com isso, esse estudante tem uma formação mais aprofundada em estudos de linguagem, o que o diferencia de um grupo que representa sujeitos não revisores. Para a escolha dos revisores, foi pré-requisito que o sujeito trabalhasse profissionalmente com revisão, em órgãos públicos, na iniciativa privada ou como autônomo. Todos os revisores tinham formação em Letras, sendo que um deles estava no último período do curso, mas já acumulava 3 anos de experiência em revisão de textos. Com relação ao tempo de experiência, 5 deles trabalhavam com revisão de 1 a 4 anos; 5, de 5 a 10 anos; e 4, por mais de 10 anos.

\subsection{Construção dos estímulos}

Os textos, do gênero jornalístico, redigidos especialmente para o experimento, consistiram de um parágrafo informativo. Os problemas dos textos experimentais foram de dois tipos:

- supressão de preposição;

- incoerência gerada por uma anáfora nominal incorreta. 
Tais tipos de problemas foram selecionados em função do custo do processamento. Esses problemas representam dois níveis diferentes: a supressão de preposição relaciona-se ao nível da palavra e do sintagma; e a incoerência gerada por uma anáfora nominal incorreta, ao nível do texto, pois é necessário que seja realizada a integração entre as sentenças do texto.

Além disso, foram inseridos textos sem erros e com outros tipos de erros, considerados distratores. Em cada sessão experimental, foram apresentados 20 textos experimentais, 10 com supressão de preposição e 10 com anáfora incorreta, nos quais não havia outros erros/ inadequações previstos de serem detectados. Além disso, havia mais 30 textos distratores ( $8 \mathrm{sem}$ erros, $2 \mathrm{com}$ um erro, $7 \mathrm{com}$ dois erros, 9 com três erros e 4 com quatro erros). ${ }^{2}$

Nos exemplos 1 e 2, abaixo, são apresentados dois exemplos de textos experimentais, um com supressão de preposição (presença duas torcidas) e outro com anáfora incorreta (americano retomando argentino).

\section{Exemplo 1 - Texto experimental - supressão de preposição}

Após um longo período, o clássico entre Atlético Mineiro e Cruzeiro contará com a presença
duas torcidas mineiras. O Cruzeiro será o mandante da partida e vai disponibilizar uma parte
dos ingressos para a torcida adversária. O jogo do Campeonato Mineiro será na próxima
semana, na inauguração do novo Mineirão, e terá policiamento reforçado.

Exemplo 2 - Texto experimental - anáfora incorreta

Na noite de ontem, um bebê foi encontrado próximo às margens da Lagoa da Pampulha por um argentino. O bebê estava enrolado em uma manta, e a mãe da criança foi presa horas depois do ocorrido. $\mathrm{O}$ americano confirmou em depoimento que a mãe abandonou a criança no local e fugiu logo em seguida.

Os textos experimentais e os distratores foram distribuídos entre 8 temas. Na construção dos textos experimentais, foram controladas variáveis que podem influenciar os resultados: 3 sentenças; 54 palavras

\footnotetext{
${ }^{2}$ Não foi feita uma distribuição em Quadrado Latino, com a leitura dos mesmos textos com e sem erros, porque isso exigiria um número maior de participantes ou mesmo um número maior de textos experimentais a serem lidos por sessão, o que seria inviável, tendo em vista a dificuldade na participação de revisores profissionais, os quais tinham que se deslocar até o local do experimento, e o cansaço gerado em função de um experimento muito longo.
} 
por texto, 18 por sentença; a posição das palavras alvo, que foram a $15^{\mathrm{a}}$ e a $16^{\mathrm{a}}$ do texto na supressão de preposição e, nos textos com anáfora incorreta, a $38^{\mathrm{a}}$, sendo que o referente incorreto era a $18^{\mathrm{a}}$ palavra do texto. Na supressão de preposição, a palavra anterior à preposição tinha 3 sílabas e a posterior, 2. Já nos textos em que havia a anáfora incorreta, tanto o referente quanto a anáfora tinham 4 sílabas.

Especificamente com relação aos textos com anáfora incorreta, levou-se em consideração, também, a estrutura informacional dos textos: o referente (item lexical novo introduzido no texto na primeira sentença) não é o sujeito da oração e vem acompanhado por um artigo indefinido. Portanto, não está focalizado. Já a anáfora incorreta é o sujeito da terceira oração e vem acompanhada por um artigo definido, com o objetivo de que se tratasse de uma retomada de um item anteriormente introduzido no discurso. Cuidou-se de compor textos distratores contendo sentenças com uma estrutura sintática semelhante, mas sem uma anáfora incorreta, de forma a minimizar as possibilidades de os participantes preverem a existência do erro em função da estrutura sintática.

Como a frequência de uso está bastante relacionada ao tempo de fixação, foi utilizado o corpus Banco de Português (BP), compilado por Tony Sardinha da PUC-SP, para se controlar essa variável. Em geral, as palavras experimentais na supressão de preposição são mais frequentes do que na anáfora incorreta.

Foi utilizado, ainda, o programa livre "Coh-Metrix-Port", ${ }^{3}$ o qual analisa vários parâmetros, visando, principalmente, oferecer subsídios para a análise da legibilidade dos textos. O índice Flesch é uma medida superficial da inteligibilidade de um texto, que leva em consideração os tamanhos médios de palavras e sentenças, correlacionando-os com a facilidade de leitura. Quanto mais alto o valor obtido, mais fácil o processamento do texto. Considerando-se esse índice, os textos do experimento são classificados como muito fáceis, fáceis ou difíceis, o que correspondente às séries anteriores ao ensino superior. Como os sujeitos participantes da pesquisa são, no mínimo, universitários, não deveriam ter dificuldade para ler o texto, levando-se em conta esse índice.

\footnotetext{
${ }^{3}$ Disponível em <www.nilc.icmc.usp.br/coh-metrix-port>. A ferramenta Coh-Metrix foi desenvolvida na Universidade de Memphis e calcula a coerência de textos, usando diversas medidas. No Brasil, a adaptação para o português foi realizada por pesquisadores do Núcleo Interistitucional de Linguística da Universidade de São Paulo - campus de São Carlos.
} 
Como a previsibilidade das palavras pode influenciar o tempo de fixação nelas, foi controlado se a palavra alvo dos textos experimentais era previsível, por meio de um teste de completação (frame), realizado com alunos do curso de Letras da UFMG. Para a supressão de preposição, a palavra alvo, ou seja, aquela posterior à preposição, é previsível, pois a palavra correta foi a preenchida entre 53 a 100\% das vezes. Já na anáfora incorreta, as palavras alvo (o referente e a anáfora) não são previsíveis, já que as escolhidas foram indicadas de 0 a $28 \%$ das vezes.

Por fim, antes do início da coleta de dados, foi realizado, um pré-teste com 16 alunos do curso de pós-graduação em Revisão de Textos da PUC-Minas, visando verificar quais erros/inadequações seriam detectados tanto nos textos experimentais quanto nos distratores. Com isso, foi possível realizar as últimas adequações nos textos experimentais, de forma a minimizar as possibilidades de os sujeitos fazerem detecções inesperadas.

\subsection{Realização da tarefa}

Para se investigar a leitura de revisores de textos profissionais, foi realizado um experimento de detecção de erros em textos jornalísticos, no qual os participantes deveriam ler e revisar os textos apresentados em uma tela de computador, clicando com o mouse nos erros/ inadequações encontrados. Foi utilizado o rastreador ocular produzido pela SR Research, modelo EyeLink 1000, disponível no Laboratório de Psicolinguística da UFMG. Esse rastreador é do tipo desktop, ou seja, a câmara e o iluminador ficam instalados abaixo do monitor de computador em que são projetados os estímulos, a uma distância de 40 a $70 \mathrm{~cm}$ da cabeça do sujeito. Foi utilizado um estabilizador de cabeça para minimizar os movimentos dos sujeitos e possibilitar o registro do reflexo pupilar e corneano. Embora o modelo de rastreador utilizado permita o registro binocular, foi realizado o registro de um dos olhos do sujeito, tendo sido definido qual deles antes do início da coleta de dados, em função da melhor calibração do equipamento.

O experimento foi realizado no Laboratório de Psicolinguística da Faculdade de Letras da UFMG. Os textos eram apresentados em uma tela de computador, aleatorizados. Primeiramente, eram apresentadas as instruções, o equipamento era calibrado e os participantes realizavam um pequeno treino de leitura e detecção de erros em três pequenos textos. Foi solicitado que os participantes lessem o texto procurando identificar 
erros e inadequações e, ao identificarem um erro/inadequação, deveriam clicar com o mouse sobre ele. Além do registro do movimento ocular, foi obtida, também, uma medida cronométrica: o tempo de resposta. Além disso, constou nas instruções que os textos foram construídos especificamente para a pesquisa e, assim, os fatos narrados não precisavam necessariamente ser verdadeiros.

Caso algum participante pedisse informações mais detalhadas sobre a atividade de revisão, o experimentador se restringia a informar que era para ele se comportar como se estivesse fazendo a revisão do texto, com a detecção de erros e inadequações. Se perguntassem quais tipos de erros deveriam ser detectados, o experimentador informava que não poderia responder, destacando que eles deveriam marcar todos os erros que encontrassem. Tal procedimento foi adotado com vistas a verificar a definição que os sujeitos tinham da tarefa de revisão, por meio dos tipos de erros detectados.

Essa revisão de cada texto deveria ser realizada em, no máximo, 1 minuto/60segundos, pois, caso se ultrapassasse esse limite de tempo, o programa passava automaticamente para o próximo texto. Entre um texto e outro, havia ainda uma tela de drift correction, em que um círculo semelhante ao da calibração era projetado na tela no exato local em que se iniciaria o próximo texto. Como o sujeito deveria fixar esse círculo para que o experimentador passasse para o próximo texto, era garantido que, no início da leitura, o sujeito estaria com o olhar fixado na primeira palavra de cada texto. Isso evita variação entre textos e sujeitos quanto ao início da leitura de cada texto. A cor de fundo era branca, a letra preta em fonte Times New Roman de tamanho 20, com espaçamento 3,5. Cuidou-se de retirar o máximo possível o brilho da tela do computador, para evitar fadiga visual.

\subsection{Análise dos dados}

Foram analisadas diversas variáveis, de vários níveis: texto, sentença e trecho alvo. Assim, a análise dos dados se pautou em dois ângulos: o nível ao qual se aplica a variável investigada (texto, sentença ou trecho) e a relação entre movimento ocular e detecção de erros pelos sujeitos.

O nível de investigação da variável tem uma relação direta com o tipo de erro investigado: a supressão de preposição, por ser um erro que tem escopo local, requer medidas que se apliquem ao nível da palavra ou 
do sintagma; já a anáfora incorreta tem escopo na integração de partes do texto e, assim, requer também medidas que correspondam aos níveis da sentença e do texto.

Além disso, neste trabalho há tanto as medidas do movimento ocular quanto a medida cronométrica de tempo de resposta. Por isso, foi possível investigar o movimento ocular e também a relação entre movimento ocular e detecção. Portanto, optou-se por utilizar medidas tradicionais nos estudos sobre leitura e movimento ocular, bem como por adaptar algumas medidas a esta pesquisa, conjugando-as com o tempo de resposta.

QUADRO 1 - Variáveis dependentes ${ }^{4}$

\begin{tabular}{|c|c|c|c|}
\hline & Texto & Sentenças & Trecho \\
\hline $\begin{array}{c}\text { Movimento } \\
\text { ocular }\end{array}$ & $\begin{array}{l}\text { - Tempo total de leitura } \\
\text { - Número de fixações } \\
\text { nos textos } \\
\text { - Duração média das } \\
\text { fixações nos textos }\end{array}$ & $\begin{array}{l}\text { - First-pass fixation } \\
\text { time nas sentenças } \\
\text { alvo } \\
\text { - Tempo total de } \\
\text { fixação nas sentenças } \\
\text { alvo } \\
\text { - Second-pass fixation } \\
\text { time nas sentenças } \\
\text { alvo } \\
\text { - Regression-path } \\
\text { reading time nas } \\
\text { sentenças alvo }\end{array}$ & $\begin{array}{l}\text { - First-pass fixation } \\
\text { time } \\
\text { - Tempo total de } \\
\text { fixação no trecho } \\
\text { alvo } \\
\text { - Número de fixações } \\
\text { no trecho alvo } \\
\text { - Regression-path }\end{array}$ \\
\hline $\begin{array}{c}\text { Movimento } \\
\text { ocular e } \\
\text { detecção }\end{array}$ & $\begin{array}{l}\text { - Tempo total de leitura } \\
\text { até a detecção } \\
\text { - Tempo total de leitura } \\
\text { após a detecção } \\
\text { - Número de fixações } \\
\text { até a detecção } \\
\text { - Número de fixações } \\
\text { após a detecção } \\
\text { - Duração média } \\
\text { das fixações até a } \\
\text { detecção } \\
\text { - Duração média das } \\
\text { fixações após a } \\
\text { detecção }\end{array}$ & $\begin{array}{l}\text { - Tempo total de } \\
\text { fixação nas sentenças } \\
\text { alvo até a detecção } \\
\text { - Tempo total de } \\
\text { fixação nas sentenças } \\
\text { alvo após a detecção } \\
\text { - Second-pass fixation } \\
\text { time nas sentenças } \\
\text { alvo até a detecção } \\
\text { - Second-pass fixation } \\
\text { time nas sentenças } \\
\text { alvo após a deteç̧ão }\end{array}$ & $\begin{array}{l}\text { - Tempo total de } \\
\text { fixação no trecho } \\
\text { alvo até a detecção } \\
\text { - Tempo total de } \\
\text { fixação no trecho } \\
\text { alvo após a detecção }\end{array}$ \\
\hline
\end{tabular}

\footnotetext{
${ }^{4}$ Neste trabalho, optou-se por manter a terminologia em inglês, uma vez que não há tradução já consagrada em português.
} 
Nas medidas agrupadas como "Movimento ocular", considerouse tanto os textos em que os erros foram detectados quanto aqueles em que eles não foram detectados, de maneira a se comparar a deteç̧ão e a não detecção. Já nas medidas agrupadas como "Movimento ocular e detecção", foram considerados somente os textos em que o erro foi detectado, uma vez que o momento da detecção (no qual o participante clicava com o mouse no erro) foi utilizado como um divisor para se analisar cada medida até e após essa detecção: por exemplo, o tempo total de leitura até a detecção do erro e o tempo total de leitura após a detecção do erro, medidas que correspondem ao tempo total de leitura até o momento em que o participante clica com o mouse sobre o erro e ao tempo total de leitura posterior a esse momento, até ele finalizar a tarefa.

O tempo total de leitura corresponde ao tempo total que o sujeito demorou lendo e revisando cada texto. Já o tempo total de leitura até a deteç̧ão do erro corresponde ao tempo que o sujeito demorou na leitura do texto até clicar com o mouse sobre o erro; e o tempo total de leitura após a detecção, ${ }^{5}$ ao tempo que o sujeito demorou desde que clicou com o mouse sobre o erro até terminar a revisão do texto. A mesma divisão aplica-se ao número de fixações no texto até e após a detecção do erro.

A duração média das fixações equivale à razão entre o tempo total de leitura, incluindo fixações e sacadas, e o número de fixações no texto. Assim, a duração média das fixações até a deteç̧ão do erro corresponde à razão entre o tempo total de leitura até a detecção e o número de fixações até a detecção. A mesma lógica aplica-se à duração média das fixações após a detecção do erro.

O first-pass fixation time corresponde à soma das durações de todas as fixações realizadas (seja na sentença alvo, quando no nível da sentença, ou na palavra alvo, quando no nível local) até seu abandono para a esquerda ou para a direita e durante a primeira leitura, ou seja, é uma medida que indica o tempo demandado em uma primeira leitura do alvo.

Já o tempo total de fixação nas sentenças equivale à soma de todas as fixações realizadas na sentença. Esse tempo também foi dividido em

\footnotetext{
${ }^{5}$ Embora já fosse esperada diferença significativa entre as condições experimentais quanto às medidas de tempo após a detecção do erro, em função da localização de cada trecho alvo (na primeira sentença no caso da supressão de preposição e na terceira para a anáfora incorreta), tais medidas foram investigadas para se analisar se os grupos fariam releituras do texto e o engajamento com a tarefa de revisão.
} 
duas partes: até e após a detecção do erro, seguindo a mesma lógica de contabilização apresentada acima.

O second-pass fixation time diz respeito à diferença entre o tempo total de fixação na sentença e ofirst-pass fixation time, ou seja, equivale ao tempo de retorno à sentença depois de ela ter sido lida uma primeira vez. Para essa medida, também foi contabilizado o valor até e após da detecção do erro.

A última medida do nível da sentença, o regression-path reading time corresponde à soma de todas as fixações realizadas pelo sujeito desde o momento em que ele fixa pela primeira vez a sentença alvo até quando ele faz uma fixação em alguma parte posterior do texto, contabilizando, além das fixações na própria sentença alvo, também as possíveis regressões realizadas a partes anteriores do texto.

O tempo total de fixação no trecho alvo diz respeito à soma de todas as fixações feitas nele, sendo que foi verificada, também, a soma de todas as fixações até a detecção do erro e após a detecção.

$\mathrm{O}$ regression-path corresponde à soma de todas as fixações realizadas pelo sujeito desde o momento em que ele fixa pela primeira vez o trecho alvo até quando ele faz uma fixação em alguma parte posterior do texto, contabilizando, além das fixações no próprio trecho alvo, também as possíveis regressões realizadas a partes anteriores do texto.

Foram variáveis independentes na análise do movimento ocular nos textos experimentais:

1. Tipos de erros:

a. supressão de preposição

b. anáfora incorreta

2. Grupos de revisão de textos:

a. revisores

b. não revisores

3. Detecção:

a. erros detectados

b. erros não detectados 
O cruzamento dessas variáveis gera 8 grupos:

1. revisores - supressão de preposição - erro detectado;

2. não revisores - supressão de preposição - erro detectado;

3. revisores - supressão de preposição - erro não detectado;

4. não revisores - supressão de preposição - erro não detectado;

5. revisores - anáfora incorreta - erro detectado;

6. não revisores - anáfora incorreta - erro detectado;

7. revisores - anáfora incorreta - erro não detectado;

8. não revisores - anáfora incorreta - erro não detectado;

Primeiramente, os dados foram tabulados e explorados descritivamente, verificando-se medidas de tendência central como média, mediana e desvio padrão, bem como com a visualização por meio de gráficos como boxplot, gráfico de barras, gráfico de densidade e de médias, escolhidos conforme o tipo de variável investigada.

Foi, então, realizada a estatística inferencial, escolhendo-se o teste estatístico mais adequado em função dos dados analisados. O nível de significância adotado foi de $95 \%$, portanto, $\alpha$ foi definido como 0,05 . O programa utilizado para a análise estatística foi o R. ${ }^{6}$

Nas variáveis em que as amostras seguiam uma distribuição normal, foi escolhido o teste paramétrico. Como o experimento foi realizado com medidas repetidas, o teste paramétrico definido foi o modelo de regressão linear misto. ${ }^{7}$

Já nas variáveis em que as amostras não seguiam uma distribuição normal, os testes não paramétricos escolhidos foram o de Kruskal-Wallis ou de Wilcoxon-Mann-Whitney, tendo em vista tratar-se de testes de postos e, assim, não ser necessário que se conheça a distribuição das amostras.

\section{Resultados}

Os revisores detectaram 191 dos 279 erros, o que corresponde a $68,5 \%$ dos erros. Já os não revisores detectaram 165 dos 280 erros,

\footnotetext{
${ }^{6}$ Disponível em <https://www.r-project.org/>.

${ }^{7}$ Este teste foi escolhido, também, porque permite analisar a relação entre as variáveis ao longo da execução da tarefa, indicando aspectos importantes do comportamento dos participantes, não explorados neste artigo, mas disponíveis em Leite (2014).
} 
o que equivale a 58,9\%. Portanto, os revisores foram mais proficientes e detectaram mais erros do que os não revisores, mas, considerando somente os textos experimentais, essa diferença não foi estatisticamente significativa.

Nas tabelas apresentadas, serão indicados os valores das médias e o t ou p valor (conforme o teste estatístico). Informações mais detalhadas, como número de valores considerados por variáveis, desvio-padrão, graus de liberdade e gráficos podem ser consultadas em Leite (2014). Os códigos utilizados em todas as tabelas apresentadas serão os seguintes:

rev: revisores

nrev: não revisores

det: erro detectado

ndet: erro não detectado

sp: supressão de preposição

an: anáfora incorreta

N/A: não se aplica

*: diferença estatisticamente significativa

. : diferença marginalmente significativa

\subsection{Nível do texto}

A seguir, são apresentadas as variáveis em que foram detectados resultados estatisticamente significativos.

Nas variáveis em que foram consideradas as medidas até e após a detecção do erro, não foram apresentados os resultados estatisticamente significativos encontrados entre os tipos de erros, ou seja, quando se comparou a anáfora incorreta e a supressão de preposição, uma vez que essas diferenças já eram esperadas em função da composição dos textos (no primeiro caso, o erro encontra-se na terceira sentença e, no segundo, na primeira). Porém, nesses casos, foram comparados os grupos de revisão em um mesmo tipo de erro (revisores na anáfora x não revisores na anáfora e revisores na supressão de preposição x não revisores na supressão de preposição). 


\section{TABELA 1 - Resumo dos valores médios dos resultados obtidos nas variáveis do nível do texto - modelos de regressão linear mistos}

\begin{tabular}{|c|c|c|c|c|c|c|c|c|c|}
\hline \multirow[t]{2}{*}{ Medida } & \multicolumn{2}{|c|}{ Média } & \multirow[t]{2}{*}{$\mathrm{t}$ valor } & \multicolumn{2}{|c|}{ Média } & \multirow[t]{2}{*}{$\mathrm{t}$ valor } & \multicolumn{2}{|c|}{ Média } & \multirow[t]{2}{*}{$\mathrm{t}$ valor } \\
\hline & rev & nrev & & an & $\mathrm{sp}$ & & det & ndet & \\
\hline $\begin{array}{c}\text { tempo total } \\
\text { de leitura } \\
\text { do texto }\end{array}$ & $35473 \mathrm{~ms}$ & $27402 \mathrm{~ms}$ & $\begin{array}{c}* \text { rev }>\text { nrev } \\
0,002\end{array}$ & $32709 \mathrm{~ms}$ & $30147 \mathrm{~ms}$ & $\begin{array}{c}* \text { an }>\text { sp } \\
0,04\end{array}$ & $31845 \mathrm{~ms}$ & $30697 \mathrm{~ms}$ & $\begin{array}{c}* \text { det }>\text { ndet } \\
0,01\end{array}$ \\
\hline $\begin{array}{c}\text { número de } \\
\text { fixações no } \\
\text { texto }\end{array}$ & 134 & 111 & $\begin{array}{c}\text { rev }>\text { nrev } \\
0,06\end{array}$ & 129 & 117 & $\begin{array}{c}* \text { an }>\text { sp } \\
0,002\end{array}$ & 123 & 123 & - \\
\hline $\begin{array}{c}\text { duração } \\
\text { média das } \\
\text { fixações no } \\
\text { texto } \\
\end{array}$ & $225 \mathrm{~ms}$ & $205 \mathrm{~ms}$ & $\begin{array}{c}\text { rev }>\text { nrev } \\
0,06\end{array}$ & $212 \mathrm{~ms}$ & $218 \mathrm{~ms}$ & $\begin{array}{c}\text { *sp>an } \\
0,000\end{array}$ & $218 \mathrm{~ms}$ & $210 \mathrm{~ms}$ & $\begin{array}{c}\text { det }>\text { ndet } \\
0,05\end{array}$ \\
\hline $\begin{array}{c}\text { tempo total } \\
\text { de leitura } \\
\text { após a } \\
\text { detecção do } \\
\text { erro } \\
\end{array}$ & $21590 \mathrm{~ms}$ & $13812 \mathrm{~ms}$ & $\begin{array}{c}* \text { rev }>\text { nrev } \\
0,001\end{array}$ & $13323 \mathrm{~ms}$ & $20696 \mathrm{~ms}$ & $\begin{array}{c}\text { *sp>an } \\
0,000\end{array}$ & N/A & N/A & N/A \\
\hline $\begin{array}{c}\text { duração } \\
\text { média das } \\
\text { fixações até } \\
\text { a detecção } \\
\text { do erro } \\
\end{array}$ & $245 \mathrm{~ms}$ & $222 \mathrm{~ms}$ & $\begin{array}{c}\text { rev>nrev } \\
0,06\end{array}$ & $219 \mathrm{~ms}$ & $243 \mathrm{~ms}$ & $\begin{array}{c}{ }_{*}^{*} \mathrm{sp}>\mathrm{an} \\
0,000\end{array}$ & N/A & N/A & $\mathrm{N} / \mathrm{A}$ \\
\hline
\end{tabular}

TABELA 2 - Resumo dos resultados de interação obtidos nas variáveis do nível do texto - modelos de regressão linear mistos

\begin{tabular}{|c|c|c|c|c|}
\hline Medida & $\begin{array}{c}\text { Variável } \\
1\end{array}$ & Variável 2 & Média (ms) & Interações (p valor) \\
\hline \multirow{4}{*}{$\begin{array}{c}\text { Número de } \\
\text { fixações no texto }\end{array}$} & \multirow[t]{2}{*}{ det } & $\mathrm{sp}$ & 104 & \multirow{4}{*}{$\begin{array}{l}* \text { an } \operatorname{det}>\operatorname{sp} \operatorname{det}(0,003) \\
* \text { an } \operatorname{det}>\text { an } n d e t(0,01)\end{array}$} \\
\hline & & an & 130 & \\
\hline & \multirow[t]{2}{*}{ ndet } & $\mathrm{sp}$ & 113 & \\
\hline & & an & 112 & \\
\hline \multirow{4}{*}{$\begin{array}{l}\text { Duração média } \\
\text { das fixações no } \\
\text { texto }\end{array}$} & \multirow[t]{2}{*}{ det } & $\mathrm{sp}$ & 214 & \multirow{4}{*}{$\begin{array}{l}* \text { sp det }>\text { an } \operatorname{det}(0,000) \\
* \text { sp det }>\text { an } n \operatorname{ndet}(0,03) \\
* \text { sp det }>\text { sp } \operatorname{ndet}(0,000)\end{array}$} \\
\hline & & an & 210 & \\
\hline & \multirow[t]{2}{*}{ ndet } & $\mathrm{sp}$ & 198 & \\
\hline & & an & 207 & \\
\hline
\end{tabular}


TABELA 3 - Resumo dos resultados obtidos no nível do texto - comparações múltiplas do teste de Wilcoxon-Mann-Withney com correção de Bonferroni

\begin{tabular}{|c|c|c|c|c|}
\hline Medida & Variável 1 & Variável 2 & Mediana & Interações (p valor) \\
\hline \multirow{4}{*}{$\begin{array}{l}\text { tempo total de } \\
\text { leitura até a } \\
\text { detecção do erro }\end{array}$} & \multirow[t]{2}{*}{$\mathrm{sp}$} & rev & $7469 \mathrm{~ms}$ & \multirow[t]{4}{*}{$*$ rev an $>$ nrev an $(0,000)$} \\
\hline & & nrev & $7645 \mathrm{~ms}$ & \\
\hline & \multirow[t]{2}{*}{ an } & rev & $22301 \mathrm{~ms}$ & \\
\hline & & nrev & $17094 \mathrm{~ms}$ & \\
\hline \multirow{4}{*}{$\begin{array}{l}\text { número de fixações } \\
\text { até a detecção do } \\
\text { erro }\end{array}$} & \multirow[t]{2}{*}{$\mathrm{sp}$} & rev & 29 & \multirow{4}{*}{$\begin{array}{l}* \text { rev an }>\text { nrev an }(0,005) \\
* \text { nrev } \mathrm{sp}>\text { rev sp }(0,01)\end{array}$} \\
\hline & & nrev & 34 & \\
\hline & \multirow[t]{2}{*}{ an } & rev & 104 & \\
\hline & & nrev & 81 & \\
\hline \multirow{4}{*}{$\begin{array}{l}\text { duração média das } \\
\text { fixações após a } \\
\text { detecção do erro }\end{array}$} & \multirow[t]{2}{*}{$\mathrm{sp}$} & rev & $276 \mathrm{~ms}$ & \multirow[t]{4}{*}{$*$ rev $\mathrm{sp}>\operatorname{nrev} \mathrm{sp}(0,02)$} \\
\hline & & nrev & $265 \mathrm{~ms}$ & \\
\hline & \multirow[t]{2}{*}{ an } & rev & $377 \mathrm{~ms}$ & \\
\hline & & nrev & $377 \mathrm{~ms}$ & \\
\hline \multirow{4}{*}{$\begin{array}{c}\text { número de fixações } \\
\text { após a detecção do } \\
\text { erro }\end{array}$} & \multirow[t]{2}{*}{$\mathrm{sp}$} & rev & 81,5 & \multirow{4}{*}{$\begin{array}{l}* \text { rev an }>\text { nrev an }(0,002) \\
* \text { rev sp }>\text { nrev sp }(0,000)\end{array}$} \\
\hline & & nrev & 56,5 & \\
\hline & \multirow[t]{2}{*}{ an } & rev & 38 & \\
\hline & & nrev & 20 & \\
\hline
\end{tabular}

Na comparação entre os dois tipos de erros, verificou-se que os sujeitos tiveram um tempo maior de leitura quando houve a anáfora incorreta, o que está associado ao número de fixações nos textos, já que, também no que se refere a essa variável, houve diferença estatisticamente significativa entre as duas condições, com maior número de fixações nos textos em que houve a anáfora incorreta. Ao contrário, as fixações foram em média mais longas nos textos em que havia a supressão de preposição, o que pôde ser evidenciado também na duração média das fixações até a detecção do erro.

Além disso, na interação entre essas variáveis (tabela 3), os resultados indicam uma diferença entre a detecção da anáfora incorreta e a detecção da supressão de preposição: enquanto na anáfora incorreta o tempo total de leitura maior é relacionado ao número maior de fixações no texto, na supressão de preposição a detecção está relacionada a fixações em média mais longas. Essa diferença de padrão, portanto, pode estar relacionada a dois tipos diferentes de leitura: uma leitura mais voltada à superfície, com fixações mais longas, e uma leitura voltada a níveis mais globais, com mais fixações no texto. 
No que se refere à análise depreendida no nível do texto, os resultados indicam que os revisores, em geral, fazem uma leitura mais demorada do texto, associada a um número maior de fixações.

$\mathrm{Na}$ comparação entre revisores e não revisores, os primeiros tiveram valores mais elevados no tempo total de leitura e tempo total de leitura após a detecção do erro (tabela 1), o que indica que, além de demandarem mais tempo lendo os textos, eles ainda mantiveram a leitura por mais tempo mesmo após terem detectado o erro. Quanto ao tempo total de leitura até a detecção do erro, quando comparados os grupos de revisão em um mesmo tipo de erro, os revisores apresentaram valores mais elevados do que os não revisores quando houve anáfora incorreta, mas não diferiram com relação à detecção da supressão de preposição (tabela 3).

Embora tenha havido uma diferença somente marginalmente significativa entre revisores e não revisores no que se refere ao número de fixações nos textos (tabela 1), ao se analisar esse número até a detecção do erro, na supressão de preposição, ele foi maior no grupo dos não revisores e, ao contrário, na anáfora incorreta, foi em média maior no grupo dos revisores. Após a detecção do erro, revisores fizeram mais fixações, independente do tipo de erro.

Quanto à duração média das fixações no texto, houve uma diferença marginalmente significativa entre revisores e não revisores, assim como quando foi analisada essa variável até a detecção do erro (tabela 1), sendo que a tendência foi de os revisores fazerem fixações em média mais longas. Houve diferença estatisticamente significativa entre os grupos no que se refere à duração média das fixações após a detecção do erro (tabela 3).

Dessa forma, os revisores mostraram um padrão de leitura diferente para cada tipo de erro: na detecção da supressão de preposição, as fixações tenderam a ser mais longas (duração média das fixações marginalmente significativa), e na da anáfora incorreta, o número de fixações foi maior.

O tempo total de leitura também foi maior nos textos em que o erro foi detectado do que naqueles em que o sujeito não detectou o erro, assim como o número de fixações, porém, tal achado pode decorrer da própria tarefa, uma vez que, nos textos em que o erro foi detectado, o participante demandou tempo clicando com o mouse no erro. 


\subsection{Nível da sentença}

Quanto às medidas apresentadas no nível da sentença, na supressão de preposição, considerou-se somente a primeira sentença, na qual estava localizado o erro. Já na anáfora incorreta, foram analisadas tanto a primeira quanto a terceira sentença, uma vez que nelas estavam localizados, respectivamente, o referente e a anáfora incorreta.

TABELA 4 - Resumo dos resultados obtidos nas variáveis do nível da sentença - modelos de regressão linear mistos

\begin{tabular}{|c|c|c|c|c|c|c|c|c|c|}
\hline \multirow[t]{2}{*}{ Medida } & \multicolumn{2}{|c|}{ Média (ms) } & \multirow[t]{2}{*}{$\mathrm{t}$ valor } & \multicolumn{2}{|c|}{ Média (ms) } & \multirow[t]{2}{*}{$\mathrm{t}$ valor } & \multicolumn{2}{|c|}{ Média (ms) } & \multirow[t]{2}{*}{$\mathrm{t}$ valor } \\
\hline & rev & nrev & & an & $\mathrm{sp}$ & & det & ndet & \\
\hline $\begin{array}{c}\text { tempo total } \\
\text { de fixação na } \\
\text { primeira sentença }\end{array}$ & 11274 & 9227 & $\begin{array}{c}* \text { rev }>\text { nrev } \\
0,02\end{array}$ & $9971 \mathrm{~ms}$ & 10527 & 0,45 & 10592 & 9646 & $\begin{array}{l}* \text { det }>\text { ndet } \\
0,000\end{array}$ \\
\hline $\begin{array}{l}\text { tempo total de } \\
\text { fixação na terceira } \\
\text { sentença }\end{array}$ & 11050 & 8240 & $\begin{array}{c}* \text { rev }>\text { nrev } \\
0,009\end{array}$ & N/A & N/A & N/A & 10756 & 8655 & $\begin{array}{l}{ }^{*} \text { det }>\text { ndet } \\
0,000\end{array}$ \\
\hline $\begin{array}{l}\text { tempo total } \\
\text { de fixação na } \\
\text { primeira sentença } \\
\text { até a detecção do } \\
\text { erro }\end{array}$ & 9075 & 8270 & $\begin{array}{c}*_{\text {rev }}>\text { nrev } \\
0,008\end{array}$ & 9378 & 8305 & 0,24 & N/A & N/A & N/A \\
\hline $\begin{array}{l}\text { tempo total de } \\
\text { fixação na terceira } \\
\text { sentença até a } \\
\text { detecção do erro }\end{array}$ & 6341 & 4868 & 0,21 & N/A & N/A & N/A & N/A & N/A & N/A \\
\hline
\end{tabular}

TABELA 5 - Resumo dos resultados de interação obtidos nas variáveis do nível da sentença - modelos de regressão linear mistos

\begin{tabular}{c|c|c|c|c}
\hline Medida & Variável 1 & Variável 2 & Média $(\mathrm{ms})$ & Interações (p valor) \\
\hline $\begin{array}{c}\text { tempo total de fixação na } \\
\text { primeira sentença até a } \\
\text { detecção do erro }\end{array}$ & sp & rev & 8192 & $*$ * rev an > nrev an $(0,03)$ \\
\cline { 2 - 4 } & an & nrev & 8431 & \multirow{2}{*}{ rev an > rev sp $(0,03)$} \\
\cline { 3 - 4 } & & rev & 10522 & \\
\cline { 3 - 4 } & & nrev & 7981 & \\
\hline
\end{tabular}


TABELA 6 - Resumo dos resultados obtidos no nível da sentença - comparações múltiplas do teste de Wilcoxon-Mann-Withney com correção de Bonferroni

\begin{tabular}{|c|c|c|c|c|c|}
\hline Medida & Variável 1 & Variável 2 & Variável 3 & $\begin{array}{l}\text { Mediana } \\
(\mathrm{ms})\end{array}$ & Interações (p valor) \\
\hline \multirow{8}{*}{$\begin{array}{l}\text { second-pass } \\
\text { fixation time na } \\
\text { primeira sentença }\end{array}$} & \multirow{4}{*}{ det } & \multirow[t]{2}{*}{$\mathrm{sp}$} & rev & 2241 & \multirow{8}{*}{$\begin{array}{l}* \text { rev an det }>\text { nrev an det }(0,000) \\
* \text { rev an det }>\text { nrev sp det }(0,000) \\
* \text { rev an det }>\text { rev sp det }(0,000) \\
* \text { rev an det }>\text { nrev an ndet }(0,000) \\
* \text { rev an det }>\text { rev an ndet }(0,000) \\
* \text { rev an det }>\text { nrev sp ndet }(0,000) \\
* \text { rev sp det }<\text { nrev sp det }(0,000) \\
* \text { nrev sp det }<\text { nrev an ndet }(0,03) \\
* \text { nrev sp det }<\text { rev an ndet }(0,03) \\
* \text { nrev sp det }<\text { rev sp ndet }(0,01)\end{array}$} \\
\hline & & & nrev & 182,5 & \\
\hline & & \multirow[t]{2}{*}{ an } & rev & 5076 & \\
\hline & & & nrev & 2088 & \\
\hline & \multirow{4}{*}{ ndet } & \multirow[t]{2}{*}{$\mathrm{sp}$} & rev & 4274 & \\
\hline & & & nrev & 1846 & \\
\hline & & \multirow[t]{2}{*}{ an } & rev & 1668,5 & \\
\hline & & & nrev & 2092 & \\
\hline \multirow{4}{*}{$\begin{array}{l}\text { first-pass fixation } \\
\text { time na terceira } \\
\text { sentença }\end{array}$} & \multirow{4}{*}{ an } & \multirow[t]{2}{*}{$\operatorname{det}$} & rev & 5338 & \multirow{4}{*}{$\begin{array}{l}* \text { nrev det }>\text { nrev ndet }(0,000) \\
* \text { nrev det }>\text { rev ndet }(0,000) \\
* \text { rev det }<\text { nrev ndet }(0,006)\end{array}$} \\
\hline & & & nrev & 6181 & \\
\hline & & \multirow[t]{2}{*}{ ndet } & rev & 6072 & \\
\hline & & & nrev & 5739 & \\
\hline \multirow{4}{*}{$\begin{array}{l}\text { second-pass } \\
\text { fixation time na } \\
\text { terceira sentença }\end{array}$} & \multirow[t]{4}{*}{ an } & \multirow[t]{2}{*}{ det } & rev & 8659 & \multirow{4}{*}{$\begin{array}{l}* \text { rev det }>\text { nrev det }(0,000) \\
* \text { rev det }>\text { nrev ndet }(0,000) \\
* \text { rev det }>\text { rev ndet }(0,000) \\
* \text { nrev det }>\text { nrev ndet }(0,000) \\
* \text { nrev det }>\text { rev ndet }(0,01)\end{array}$} \\
\hline & & & nrev & 6039 & \\
\hline & & \multirow[t]{2}{*}{ ndet } & rev & 2118,5 & \\
\hline & & & nrev & 1666 & \\
\hline \multirow{4}{*}{$\begin{array}{l}\text { regression-path } \\
\text { reading time na } \\
\text { terceira sentença }\end{array}$} & \multirow[t]{4}{*}{ an } & \multirow[t]{2}{*}{$\operatorname{det}$} & rev & 4223,5 & \multirow{4}{*}{$\begin{array}{l}* \text { rev det }>\text { nrev ndet }(0,000) \\
* \text { rev det }>\text { rev ndet }(0,000) \\
* \text { nrev det }>\text { rev ndet }(0,002) \\
* \text { nrev det }>\text { nrev ndet }(0,000)\end{array}$} \\
\hline & & & nrev & 4282 & \\
\hline & & \multirow[t]{2}{*}{ ndet } & rev & 2489,5 & \\
\hline & & & nrev & 2199 & \\
\hline \multirow{4}{*}{$\begin{array}{l}\text { tempo total } \\
\text { de fixação na } \\
\text { primeira sentença } \\
\text { após a detecção } \\
\text { do erro }\end{array}$} & \multirow[t]{4}{*}{ det } & \multirow[t]{2}{*}{ sp } & rev & 2208,5 & \multirow{4}{*}{$\begin{array}{l}* \text { rev an }>\text { nrev an }(0,001) \\
* \text { rev an }<\text { rev sp }(0,000) \\
* \text { nrev an }<\operatorname{nrev} s p(0,000) \\
* \text { nrev an }<\operatorname{rev} \mathrm{sp}(0,000) \\
* \text { rev sp }>\text { nrev sp }(0,000)\end{array}$} \\
\hline & & & nrev & 525,5 & \\
\hline & & \multirow[t]{2}{*}{ an } & rev & 506,0 & \\
\hline & & & nrev & 0 & \\
\hline \multirow{2}{*}{$\begin{array}{c}\text { tempo total de } \\
\text { fixação na terceira } \\
\text { sentença após a } \\
\text { detecção }\end{array}$} & \multirow[t]{2}{*}{ det } & an & rev & 5232 & $*$ rev $>$ nrev $(0,000)$ \\
\hline & & & nrev & 2933 & \\
\hline second-pass & det & $\mathrm{sp}$ & rev & 0 & $*$ rev an $>$ nrev an $(0,02)$ \\
\hline $\begin{array}{l}\text { fixation time na } \\
\text { primeira sentenca }\end{array}$ & & & nrev & 0 & $\begin{array}{l}* \text { rev an }>\text { rev sp }(0,000) \\
* \text { rev an }>\text { nrev sp }(0,000)\end{array}$ \\
\hline até a detecção & & an & rev & 2904,5 & * nrev an > rev sp $(0,000)$ \\
\hline & & & nrev & 1526 & $*$ nrev an $>$ nrev sp $(0,000)$ \\
\hline
\end{tabular}

Quando foram comparados revisores e não revisores, houve diferença estatisticamente significativa quanto ao tempo total de fixação na primeira e na terceira sentenças do texto, quanto ao tempo total de fixação na primeira sentença do texto até a detecção do erro, bem como quanto ao tempo total de fixação na primeira e na terceira sentenças após 
a detecção do erro. Em todas essas situações, seja na anáfora incorreta ou na supressão de preposição, os revisores apresentaram valores em média mais elevados do que os não revisores. Os dois grupos também diferiram quanto ao second-pass fixation time na primeira e na terceira sentença, sendo que os revisores tiveram um tempo de retorno a essas sentenças maior do que os não revisores. Os revisores também tiveram um tempo de retorno à primeira sentença até a detecção da anáfora incorreta (second-pass fixation time até a detecção do erro) maior do que os não revisores, mas os dois grupos não diferiram no que se refere à supressão de preposição, pois, em ambos, a detecção da supressão de preposição ocorreu na primeira leitura da sentença.

\subsection{Nível local}

Na condição de supressão de preposição, foi analisado somente o tempo total de fixação no trecho alvo, que abarca a $15^{\mathrm{a}}$ e a $16^{\mathrm{a}}$ palavra do texto. Já na condição de anáfora incorreta, foi analisado o tempo total de fixação no referente e na anáfora, em separado.

TABELA 7 - Resumo dos resultados obtidos nas variáveis do nível local modelos de regressão linear mistos

\begin{tabular}{|c|c|c|c|c|c|c|c|c|c|}
\hline \multirow[t]{2}{*}{ Medida } & \multicolumn{2}{|c|}{ Média } & \multirow[t]{2}{*}{$\mathrm{t}$ valor } & \multicolumn{2}{|c|}{ Média } & \multirow[t]{2}{*}{$\mathrm{t}$ valor } & \multicolumn{2}{|c|}{ Média } & \multirow[t]{2}{*}{$\mathrm{t}$ valor } \\
\hline & rev & nrev & & an & $\mathrm{sp}$ & & $\operatorname{det}$ & ndet & \\
\hline $\begin{array}{l}\text { número de } \\
\text { fixações no } \\
\text { alvo - sp }\end{array}$ & 10,74 & 11,74 & 0,16 & $\mathrm{~N} / \mathrm{A}$ & N/A & N/A & 11,91 & 7,68 & $\begin{array}{l}* \text { det }>\text { ndet } \\
0,000\end{array}$ \\
\hline $\begin{array}{l}\text { tempo total } \\
\text { de fixação no } \\
\text { referente até } \\
\text { a detecção }\end{array}$ & $1169 \mathrm{~ms}$ & $831 \mathrm{~ms}$ & $\begin{array}{c}* \text { rev }>\text { nrev } \\
0,007\end{array}$ & N/A & N/A & N/A & N/A & N/A & N/A \\
\hline
\end{tabular}

TABELA 8 - Resumo dos resultados de interação obtidos nas variáveis do nível local - modelos de regressão linear mistos

\begin{tabular}{|c|c|c|c|c|}
\hline Medida & Variável 1 & Variável 2 & Média & Interações ( $p$ valor) \\
\hline \multirow{4}{*}{$\begin{array}{l}\text { número de } \\
\text { fixações no } \\
\text { alvo - sp }\end{array}$} & \multirow[t]{2}{*}{$\operatorname{det}$} & rev & 11 & \multirow{4}{*}{$\begin{array}{l}* \text { rev det }>\text { rev ndet }(0,000) \\
* \text { rev det }>\text { nrev ndet }(0,000) \\
* \text { nrev det }>\text { rev ndet }(0,000) \\
* \text { nrev det }>\text { rev ndet }(0,000)\end{array}$} \\
\hline & & nrev & 13 & \\
\hline & \multirow[t]{2}{*}{ ndet } & rev & 8 & \\
\hline & & nrev & 7 & \\
\hline
\end{tabular}


TABELA 9 - Resumo dos resultados obtidos no nível local - comparações múltiplas do teste de Wilcoxon-Mann-Withney com correção de Bonferroni

\begin{tabular}{|c|c|c|c|c|}
\hline Medida & Variável 1 & Variável 2 & Mediana & Interações (p valor) \\
\hline \multirow{4}{*}{$\begin{array}{l}\text { tempo total } \\
\text { de fixação no } \\
\text { alvo }-\mathrm{sp}\end{array}$} & \multirow[t]{2}{*}{ det } & rev & $2980 \mathrm{~ms}$ & \multirow{4}{*}{$\begin{array}{l}* \text { nrev det }>\text { nrev ndet }(0,000) \\
* \text { nrev det }>\text { rev ndet }(0,000) \\
* \text { rev det }>\text { nrev ndet }(0,000) \\
* \text { rev det }>\text { rev ndet }(0,000)\end{array}$} \\
\hline & & nrev & $3233 \mathrm{~ms}$ & \\
\hline & \multirow[t]{2}{*}{ ndet } & rev & $1072 \mathrm{~ms}$ & \\
\hline & & nrev & $1085 \mathrm{~ms}$ & \\
\hline \multirow{4}{*}{$\begin{array}{l}\text { tempo total } \\
\text { de fixação no } \\
\text { referente }\end{array}$} & \multirow[t]{2}{*}{ det } & rev & $1229 \mathrm{~ms}$ & \multirow{4}{*}{$\begin{array}{l}* \text { rev det }>\text { nrev det }(0,002) \\
* \text { nrev det }>\text { nrev ndet }(0,008) \\
* \text { rev det }>\text { nrev ndet }(0,000) \\
* \text { rev det }>\text { rev ndet }(0,000)\end{array}$} \\
\hline & & nrev & $726 \mathrm{~ms}$ & \\
\hline & \multirow[t]{2}{*}{ ndet } & rev & $724,5 \mathrm{~ms}$ & \\
\hline & & nrev & $497 \mathrm{~ms}$ & \\
\hline \multirow{4}{*}{$\begin{array}{c}\text { tempo total } \\
\text { de fixação na } \\
\text { anáfora }\end{array}$} & \multirow[t]{2}{*}{ det } & rev & $2092 \mathrm{~ms}$ & \multirow{4}{*}{$\begin{array}{l}* \text { rev det }>\text { nrev ndet }(0,000) \\
* \text { rev det }>\text { rev ndet }(0,000) \\
* \text { nrev det }>\text { nrev ndet }(0,000) \\
* \text { nrev det }>\text { rev ndet }(0,000) \\
\text { rev det }>\text { nrev det }(0,06)\end{array}$} \\
\hline & & nrev & $1799 \mathrm{~ms}$ & \\
\hline & \multirow[t]{2}{*}{ ndet } & rev & $669 \mathrm{~ms}$ & \\
\hline & & nrev & $475 \mathrm{~ms}$ & \\
\hline \multirow{4}{*}{$\begin{array}{l}\text { número de } \\
\text { fixações no } \\
\text { referente }\end{array}$} & \multirow[t]{2}{*}{ det } & rev & 6 & \multirow{4}{*}{$\begin{array}{l}* \text { nrev det }>\text { nrev ndet }(0,003) \\
* \text { rev det }>\text { nrev ndet }(0,000) \\
* \text { rev det }>\text { rev ndet }(0,000) \\
\text { rev det }>\text { nrev det }(0,05)\end{array}$} \\
\hline & & nrev & 4 & \\
\hline & \multirow[t]{2}{*}{ ndet } & rev & 3 & \\
\hline & & nrev & 3 & \\
\hline \multirow{4}{*}{$\begin{array}{l}\text { número de } \\
\text { fixações na } \\
\text { anáfora }\end{array}$} & \multirow[t]{2}{*}{ det } & rev & 8 & \multirow{4}{*}{$\begin{array}{l}* \text { nrev det }>\text { nrev ndet }(0,000) \\
* \text { rev det }>\text { nrev ndet }(0,000) \\
* \text { rev det }>\text { rev ndet }(0,000) \\
* \text { nrev det }>\text { rev ndet }(0,000) \\
\text { rev det }>\text { nrev det }(0,05)\end{array}$} \\
\hline & & nrev & 7 & \\
\hline & \multirow[t]{2}{*}{ ndet } & rev & 3 & \\
\hline & & nrev & 2 & \\
\hline \multirow{2}{*}{$\begin{array}{c}\text { tempo total } \\
\text { de fixação no } \\
\text { alvo após a } \\
\text { detecção }- \text { sp }\end{array}$} & \multirow[t]{2}{*}{ det } & rev & $675,5 \mathrm{~ms}$ & \multirow[t]{2}{*}{$* \operatorname{rev}>\operatorname{nrev}(0,000)$} \\
\hline & & nrev & $104 \mathrm{~ms}$ & \\
\hline \multirow{2}{*}{$\begin{array}{l}\text { tempo total } \\
\text { de fixação na } \\
\text { anáfora após } \\
\text { a detecção }\end{array}$} & \multirow[t]{2}{*}{ det } & rev & $246,5 \mathrm{~ms}$ & \multirow[t]{2}{*}{$*$ rev > nrev $(0,000)$} \\
\hline & & nrev & $0 \mathrm{~ms}$ & \\
\hline
\end{tabular}

Quando foi verificado o tempo total de fixação no trecho alvo durante toda a leitura do texto, sem se levar em consideração o tempo da detecção, não foram encontradas diferenças estatisticamente significativas entre os revisores e não revisores. Ao contrário, houve diferenças estatisticamente significativas entre os textos em que os erros foram detectados e aqueles em que os erros não foram detectados, 
sendo que, quando houve a detecção, o tempo total de fixação no alvo foi maior. Esse padrão foi encontrado nos textos em que havia supressão de preposição e também naqueles em que havia a anáfora incorreta. Quanto a esse tipo de erro, somente no referente houve diferença entre revisores e não revisores, com valores mais elevados entre os primeiros. Os mesmos resultados foram obtidos na análise do número de fixações no alvo, mas, nesse caso, a diferença entre revisores e não revisores quanto ao número de fixações no referente e na anáfora foi apenas marginalmente significativa.

Da mesma forma, ao se analisar o tempo total de fixação no alvo até a detecção do erro, ou seja, a soma de todas as fixações realizadas no alvo até que o participante clicasse no mouse, inclusive os retornos feitos na palavra até então, houve diferença significativa entre revisores e não revisores somente quanto ao referente, na anáfora incorreta, sendo que, nesse caso, os valores em geral mais elevados foram encontrados entre os revisores. $\mathrm{Na}$ análise do tempo total de fixação no referente após a detecção, ou seja, depois de terem clicado com o mouse no erro, porém, não houve diferença significativa entre revisores e não revisores, já que ambos os grupos, na maioria das vezes, não retornaram ao referente.

Já no que se refere à região da anáfora incorreta, houve diferença estatisticamente significativa entre revisores e não revisores quanto ao tempo total de fixação após a detecção do erro. Portanto, os revisores demandaram mais tempo fixando o referente para detectarem a incongruência e, possivelmente, buscar resolvê-la. Depois de detectarem o erro, eles ainda fizeram mais retornos à anáfora, antes de finalizarem a leitura do texto.

$\mathrm{Na}$ supressão de preposição, como ocorreu com o tempo total de fixação no alvo, também não houve diferença estatisticamente significativa entre revisores e não revisores quanto ao tempo total de fixação no alvo até a detecção do erro. O oposto ocorreu quanto ao tempo total de fixação no alvo após a detecção do erro: os revisores tiveram valores, em média, mais elevados do que os não revisores.

Dessa forma, embora não tenha havido diferença significativa entre os revisores e os não revisores ao se analisar o tempo total de fixação no alvo na leitura do texto, essa diferença surgiu ao se analisar o movimento ocular levando-se em conta o tempo de detecção do erro. Nesse caso, os revisores, em geral, tiveram valores mais elevados do que os não revisores e, nos textos em que os erros foram detectados, os 
valores de tempo total de fixação no alvo foram mais elevados do que nos textos em que os erros não foram detectados. Essa variável, portanto, é relevante ao se investigar a proficiência na detecção dos erros.

\section{Considerações finais}

Os resultados indicaram que, em geral, os textos em que houve a anáfora incorreta apresentaram valores mais elevados nas medidas investigadas, em especial do nível do texto, inclusive com um tempo total de leitura maior, relacionado com um maior número de fixações. Esse resultado corrobora os estudos da área, indicando que erros de níveis que exigem a integração de partes dos textos acarretam mais dificuldade no processamento. A exceção foi a duração média das fixações no texto e, também, até a detecção do erro. Nesse caso, os valores foram mais elevados nos textos em que havia a supressão de preposição. Como nessa medida a interação entre o tipo de erro e a detecção foi significativa, pode-se dizer que o aumento da duração média das fixações no texto, inclusive no início, até a detecção, favorece a identificação da supressão de preposição. Tendo em vista a localização dos erros nos textos e a complexidade deles, essas diferenças podem decorrer das estratégias globais utilizadas na leitura numa fase inicial ou mais tardia. Por isso, é importante que outros estudos explorem esse achado inicial, inclusive comparando uma condição de base, com e sem erro.

Os resultados desta pesquisa corroboram o proposto por Vauras, Hyona e Niemi (1992), segundo os quais as incoerências aumentam tanto o tempo de fixação quanto o número de regressões. Esta pesquisa, portanto, confirmou dados da área de Linguística que diferenciam uma leitura voltada para níveis mais globais de uma leitura voltada para níveis mais superficiais, contribuindo, também, para a caracterização desses tipos de leitura, assim como para mostrar quais estratégias podem ser mais indicadas para cada nível. Nesse sentido, um achado importante, porém inicial, foi que a detecção da supressão de preposição é favorecida quando são realizadas fixações em média mais longas. Muitos estudos da área propõem que a leitura voltada para níveis superficiais deve ser diferente daquela voltada para níveis mais globais, sem indicarem, porém, como essa diferença se processa quanto ao movimento dos olhos. A duração mais longa das fixações parece ser um parâmetro significativo, sendo necessários outros estudos, inclusive com diferentes tipos de erros de superfície, para confirmar esse achado. 
Com relação às diferenças entre os textos em que o erro foi detectado e aqueles em que o erro não foi detectado, a perspectiva geral é de que a detecção do erro é acompanhada pelo aumento do tempo de fixação, seja no nível do texto, da sentença ou local. No nível local, nos textos em que o erro é detectado, o tempo total de fixação no alvo é maior, o que ocorre tanto na supressão de preposição quanto na anáfora incorreta. Tal diferença, porém, pode ser decorrente do tempo demandado pelos participantes para clicar com o mouse no texto.

Por fim, no que se refere à diferença entre revisores e não revisores, a perspectiva geral foi de que os revisores apresentaram valores mais elevados nas variáveis em que houve diferença significativa, o que significa que eles foram mais lentos na leitura do que os não revisores. Isso ocorreu no nível do texto, da sentença e no nível local.

Os resultados, portanto, confirmaram a hipótese inicial de que os revisores profissionais teriam uma leitura mais demorada, menos automática, mais detalhada e controlada. De acordo com Hayes (2004), com a prática extensiva, certos aspectos da edição podem se tornar automáticos, mas não a atividade de edição como um todo, mesmo em sujeitos com muita experiência, motivo pelo qual o controle da leitura é um pressuposto da revisão. Klein e Hoffman (1992), ao discorrerem sobre a expertise, destacam que a performance diferenciada de um expert pode ser identificada por diversos fatores: variabilidade-consistência, acurácia, completude e velocidade. Quanto à velocidade, os autores destacam que, em alguns casos, experts podem ser mais demorados do que novatos no cumprimento de uma tarefa, inclusive quando os novatos, por impulsividade, adotam um comportamento muito rápido. Esta pesquisa indica a ocorrência desse tipo de situação.

Os revisores profissionais também foram mais específicos quanto aos retornos realizados. Nos textos em que havia a anáfora incorreta, tiveram um tempo total de fixação na primeira sentença até a detecção do erro maior do que os não revisores e, também, um tempo total de fixação maior no referente. Como o comportamento geral dos sujeitos, ao se depararem com a anáfora incorreta, foi retornar a partes anteriores do texto, os revisores foram mais específicos e fixaram por mais tempo a parte do texto que era realmente importante para a resolução do problema, ou seja, o referente e a sentença na qual ele está contido. Além disso, após detectarem a anáfora incorreta, os revisores fizeram retornos mais longos à terceira sentença do texto e, também, à anáfora em si, o que 
indica que, também neste caso, eles foram mais específicos. De acordo com Hyona e Nurminem (2006), leitores proficientes tendem a direcionar suas regressões a partes realmente informativas do texto.

Por fim, deve-se considerar que esta pesquisa investigou especificamente a detecção de erros, que é uma das etapas da revisão, sendo que, além dela, é necessário também realizar as correções, etapa esta que não foi investigada neste momento, mas que merece consideração em estudos posteriores da área, inclusive naqueles em que se verifique a qualidade das correções realizadas, de cunho, portanto, qualitativo. Tendo em vista que os estudos sobre a revisão de textos profissional são escassos, principalmente com abordagem cognitiva, essa pesquisa teve um caráter exploratório e trouxe considerações iniciais importantes não só para o estudo da performance de revisores profissionais, mas também para o estudo do processo de leitura e revisão de textos.

\section{Referências}

ALAMARGOT, D. et al. Eye and Pen: a new device to study reading during writing. Behaviour Research Methods, Instruments and Computers, Bethesda, USA, v. 38, n. 2, p. 287-299, 2006. doi.org/10.3758/ BF03192780.

HAYES J. R. A new framework for understanding cognition and affect in writing. In: C. Levy; S. Ransdell (Ed.). The science of writing: theories, methods, individual differences and applications. Mahwah: Lawrence Erlbaum Associates, 1996. p. 1-28.

HAYES, J. R. What triggers revision? In: ALLAL, L.; CHANQUOY, L.; LARGY, P. (Ed.). Revision: cognitive and instructional processes. Kluwer Academic Publishers, Masssachusetts, 2004, p. 9-22. doi.org/10.1007/97894-007-1048-1_2.

HAYES J. R. et al. Cognitive processes in revision. In: ROSENBERG, S. (Ed.). Advances in psycholinguistics: Cambridge: Cambridge University Press, 1987. v. II: Reading, writing, and language processing, p. 176-240.

HAYES, J. R.; FLOWER, L. S. Identifying the organization of writing processes. In: GREGG, L. W.; STEINBERG, E. R. (Ed.). Cognitive processes in writing: An interdisciplinary approach. Lawrence Erlbaum Associates, Hillsdale, 1980. p. 3-30. 
HEURLEY, L. La révision de texte: L'approche de la Psychologie Cognitive. Langages, Larousse, v. 164, n. 4, p. 10-25, 2006. doi.org/10.3917/ lang.164.0010.

HYONA, Jukka; KAAKINEN, Johanna K.; LORCH, Robert F. Jr. Individual Differences in Reading to Summarize Expository Text: Evidence From Eye Fixation Patterns. Journal of Educational Psychology, Taylor \& Francis, v. 94, n. 1, p. 44-55, 2002. doi.org/10.1037/00220663.94.1.44.

HYONA, Jukka; NIEMI, Pekka. Eye Movements During Repeated Reading of a Text. Acta Psychologica, Elsevier, v. 73, p. 259-280, 1990. doi.org/10.1016/0001-6918(90)90026-C.

HYONA, Jukka; NURMINEN, Anna-Mari. Do adult readers know how they read? Evidence from eye movement patterns and verbal reports. British Journal of Psychology, John Wiley \& Sons, v. 97, p. 31-50, 2006. doi.org/10.1348/000712605X53678.

KLEIN, G. A.; HOFFMAN, R. R. Seeing the invisible: perceptualcognitive aspects of expertise. In: RABINOWITZ, M. (Ed.). Cognitive Science Foundations of Instruction. Mahwah, N. J: Eribaum, 1992. p. 203-226.

LAND, Michael F. Fixation Strategies during active behavior: a brief history. In: VAN GOMPEL, R. P. G. et al. (Ed.). Eye Movements: a window on mind and brain. Amsterdã: Elsevier, 2007. p. 76-95.

LEITE, Délia Ribeiro. O olhar do profissional: estudo do movimento ocular na leitura realizada por revisores de textos. 2014. Tese (Doutorado) - Universidade Federal de Minas Gerais, Belo Horizonte, 2014.

LUEGI, Paula. O registro do movimento dos olhos durante a leitura de textos. Dissertação de mestrado. Universidade de Lisboa, 2006.

RAYNER, Keith. Eye Movements in Reading and Information Processing: 20 Years of Research. Psychological Bulletin, Washington, v. 124, n. 3, p. 372-422, 1998. doi.org/10.1037/0033-2909.124.3.372.

STAUB, Adrian; RAYNER, Keith. Eye movements and on-line comprehension processes. In: GASKELL, G. (Ed.). The Oxford Handbook of Psycholinguistics. Oxford, UK: Oxford University Press, 2007. p. 327-342. 
VAURAS, Marja; HYONA, Jukka; NIEMI, Pekka. Comprehending coherent and incoherent texts: evidence from eye movement patterns and recall performance. Journal of Research in Reading, Wiley online Library, v. 15, n. 1, p. 39-54, 1992. doi.org/10.1111/j.1467-9817.1992.tb00020.x. WAGNER, Luiz Roberto; CUNHA, Djenane S. W. Categorias de um revisor. Língua Portuguesa - conhecimento prático, 2012. n. 37, p. 12-13. 